\title{
Paul Tholey (2018): Gestalttheorie von Sport, Klartraum und Bewusstsein. Ausgewählte Arbeiten, herausgegeben und eingeleitet von Gerhard Stemberger. Wien: Krammer Verlag, 310 Seiten, $€ 36$. ISBN: 978-3-901811-76-0.
}

Paul Tholey (1937-1998) ist für mich nicht nur ein exzellenter Vertreter der Gestalttheorie, der grundlegende Arbeiten zu Bewusstseinsphänomenen veröffentlicht und der als Pionier der Klartraumforschung weltweit Ansehen erworben hat, sondern er hatte auch wie wenige die Fähigkeit, den erkenntnistheoretischen Ansatz der Gestalttheorie, den Kritischen Realismus, präzise und - auch einem Laienpublikum - verständlich zu beschreiben. Darüber hinaus gelang es ihm, die herausragende Bedeutung dieses Ansatzes für das Verständnis verschiedenster Bewusstseinsvorgänge abzuleiten und darzustellen.

In diesem erfreulicherweise nun vorliegenden Band befinden sich die wohl wichtigsten Arbeiten von Paul Tholey aus den Jahren 1977 bis 1989 zu den Themenbereichen Sensumotorik, Bewegung und Sport, Klartraum und Bewusstseinsveränderung, Gestaltpsychologie und Phänomenologie. Dabei sind neben den in der Gestalt Theory erschienenen Beiträgen (vor allem zur Klartraumtechnik) auch bislang nur schwer zugängliche oder längst vergriffene Veröffentlichungen. Besonders erfreulich finde ich, dass die beiden wichtigen Tholey-Artikel aus der Zeitschrift BEWUSST SEIN, die bei ihrem Erscheinen nur einen kleinen Leserkreis erreichten, in diesem Band enthalten sind und es damit möglich wird, sie wieder einem größeren Leserkreis zur Verfügung zu stellen.

Der Sammelband vereint elf Arbeiten von Paul Tholey, die von Gerhard Stemberger in die drei Themenbereiche „Gestalttheorie von Sensumotorik, Bewegung und Sport", „Gestalttheorie des Klartraums und der Bewusstseinsveränderung" und „Zur Gestaltpsychologie und Phänomenologie“ aufgeteilt werden.

In seiner sehr lesenswerten Einleitung stellt Gerhard Stemberger die elf Artikel Tholeys in einer Übersicht vor und vermittelt dem Leser bereits die ersten Einsichten in die von Tholey formulierten Erkenntnisse, sowie deren Bedeutung für die Weiterentwicklung der Gestalttheorie und damit auch für die Gestalttheoretische Psychotherapie. Die Denk- und Arbeitsweise Paul Tholeys, sowie seine wissenschaftliche Intention und sein gesellschaftspolitischer Anspruch treten klar hervor und regen an, sich intensiver mit diesen Texten zu beschäftigen. 


\section{Gestalttheorie von Sensumotorik, Bewegung und Sport}

Dieser Abschnitt besteht aus drei Arbeiten, die in einem engen Zusammenhang stehend verstanden werden können. In „Erkenntnistheoretische und systemtheoretische Grundlagen der Sensumotorik“ (1980), „Sensumotorisches Lernen als Organisation des psychischen Gesamtfeldes“ (1984) und „Prinzipien des Lehrens und Lernens sportlicher Handlungen aus gestalttheoretischer Sicht" (1987) beschreibt Tholey ausführlich und umfassend die Erkenntnistheorie der Gestalttheorie, den Kritischen Realismus mit der klaren Trennung zwischen physikalischer und phänomenaler - also erlebter - Welt, der ihm stets als theoretisches Fundament für seine weiteren Schlussfolgerungen dient. Er zeigt auf, dass sensumotorische Vorgänge und das Gelingen oder Scheitern von Bewegungen erklärt werden können, wenn wir sie als ein ständiges Rückkoppelungssystem in unserer phänomenalen Wahrnehmungswelt verstehen. Welche praktischen Folgerungen sich daraus ergeben können, beschreibt er sehr eindrücklich am Beispiel des Erlernens von sportlichen Fertigkeiten, bei denen unsere Wahrnehmungswelt eine entscheidende Rolle spielen kann. Z.B. kann die Vorstellung des Skifahrers, die Skier wären nicht Sportgeräte außerhalb seines Körpers, sondern mit seinem Körper verwachsen, also Teil des erlebten Körpers, die Bewegungsabläufe des Skifahrers wesentlich verändern und erleichtern. Dass derartige Erkenntnisse nicht nur für das Erlernen von sportlichen Fähigkeiten dienlich sind, sondern darüber hinaus in vielen Lebenslagen von Bedeutung sein können (hier könnte auch Behindertenarbeit genannt werden), belegt die weiten Anwendungsmöglichkeiten der Forschungsergebnisse von Paul Tholey in diesem Feld.

\section{Gestalttheorie des Klartraums und der Bewusstseinsveränderung}

In sechs Beiträgen werden die theoretische Begründung und die Forschungsergebnisse Tholeys zum Klartraum und zur Klartraumtechnik dargestellt und nachvollzogen. „Der Klartraum. Seine Funktion in der experimentellen Traumforschung“ (1977), „Klarträume als Gegenstand empirischer Untersuchungen“ (1980), „Empirische Untersuchungen über Klarträume“ (1981), „Haben Traumgestalten ein eigenes Bewusstsein? Eine experimentell-phänomenologische Klartraumstudie" (1985), "Bewusstsein, Bewusstseinsforschung, Bewusst Sein" (1989) und „Die Entfaltung des Bewusstseins als ein Weg zur schöpferischen Freiheit - Vom Träumer zum Krieger" (1989) gelten als ganz wesentliche Beiträge zur Klartraumforschung und zur zunehmenden Differenzierung in der Entwicklung dieses Forschungsgegenstandes. In ihnen entwickelt Tholey einen eigenen Zugang zum Verständnis des Traumes und des Träumens. Stets bezieht er sich in seinen Überlegungen auf den Kritischen Realismus, mit dessen Grundlagen er eine hilfreiche Beschreibung des Unterschiedes zwischen Wach- und Traumwirklichkeit entwickelt und darüber hinaus verschiedene Bewusstseinszustände 
verständlich macht, die für ihn bei der Entwicklung eines freien Menschen in einer freien Gesellschaft von Bedeutung sein können. Besonders in seinem letzten Beitrag beschäftigt er sich mit der Entfaltung des Bewusstseins und einer Persönlichkeitsentwicklung, die von einer egozentrischen zu einer mitweltbezogenen Sicht- und Lebensweise führen kann. Darin verdeutlichen sich auch seine gesellschaftspolitischen Forderungen, die ihm bei seiner wissenschaftlichen Tätigkeit stets ein großes Anliegen waren.

\section{Zur Gestaltpsychologie und Phänomenologie}

In zwei Beiträgen „Gestaltpsychologie“ (1980) und „Deshalb Phänomenologie! Anmerkungen zur experimentell-phänomenologischen Methode“ (1986) verdeutlicht Tholey seinen wissenschaftlichen und theoretischen Standpunkt. Beim ersten Beitrag handelt es sich um eine beeindruckende und differenzierte Beschreibung der Gestaltpsychologie, die ihm bei all seinen Forschungen als Grundlage dient. Der zweite Beitrag enthält eine ausführliche Darstellung und Begründung des von ihm vertretenen phänomenologisch-experimentellen Ansatzes, womit er auch nochmals belegt, was ihn in seinen in diesem Band veröffentlichten Arbeiten zu den verschiedenen Themenbereichen als wissenschaftliche Orientierung angeleitet hat.

Es ist ein großer Verdienst Gerhard Stembergers, diese elf wichtigen Beiträge Paul Tholeys in einem Band zusammengestellt und dem interessierten Leser wieder zugänglich gemacht zu haben. Auch wenn sie bereits vor Jahren erstmals veröffentlicht wurden, so haben die beschriebenen Forschungsansätze und -ergebnisse nichts an Aktualität verloren und gehören weiterhin zur Standardliteratur der Gestalttheorie. Die Texte sind gleichzeitig Nachschlagwerk wie auch stete Herausforderung nach wiederholter und vertiefter Lektüre, um sich selbst immer wieder mit den gestalttheoretischen Konzepten auseinanderzusetzen und den Wert dieses theoretischen Ansatzes ausschöpfen zu können.

\section{Rainer Kästl, Lindau}

Rainer Kästl, geb. 1949, Dipl. Psych., Psychotherapeut (Gestalttheoretische Psychotherapie, Integrative Gestalttherapie) und Supervisor in Lindau/Bodensee und Wien, mehrere psychotherapeutische Aus- und Weiterbildungen. Lehrtherapeut in der österreichischen Arbeitsgemeinschaft für Gestalttheoretische Psychotherapie (ÖAGP). Veröffentlichungen zu den Grundkonzepten Gestalttheoretischer Psychotherapie.

Adresse: Oberer Schrannenplatz 4, D-88131 Lindau/Bodensee, Deutschland

E-mail: praxis-kaestl@t-online.de 